\title{
Spatial Neutralization of Intense Electromagnetic Waves Noise
}

\section{Souhir Tounsi}

National School of Electronics and Telecommunications of Sfax, SETIT-Research Unit, Sfax University, Sfax, Tunisia

Email address:

souhir.tounsi@enetcom.rnu.tn (S. Tounsi)

To cite this article:

Souhir Tounsi. Spatial Neutralization of Intense Electromagnetic Waves Noise. International Journal of Electrical Components and Energy Conversion. Vol. 2, No. 2, 2016, pp. 8-14. doi: 10.11648/j.ijecec.20160202.11

Received: March 28, 2016; Accepted: April 10, 2016; Published: June 1, 2016

\begin{abstract}
In this paper we present a methodology for the design and control of a neutralizer to reduce pollution of the space with not useful electromagnetic waves. Thereby innovated structure of intense wave neutralizer is presented. Sizing and modelling program is developed. This study is completed by the implementation of a control model adapted to the neutralizer sizing program. The simulation results obtained are with good scientific level and promotes the industrialization of the overall system studied.
\end{abstract}

Keywords: Location, Identification, Neutralization, Design, Control, Simulations

\section{Introduction}

The excessive use of the Internet and distance communication systems such as electrical systems operating at high current and high frequency make the space environment affected by electromagnetic resonances by also by the propagation of intense electromagnetic waves may seriously interfere with the health of the human being as the proper functioning of electrical and electronic systems. These problems can cause dryness of the skin of humans, fatal heart attacks is another serious disease. Moreover, these electromagnetic noises can disrupt the normal functioning of electrical and electronic systems and eventually lead, for example to serious machine accidents and electrical network disturbances that can make the life of the human being annoying. Therefore, we will study an intense electromagnetic wave neutralization system based on the generation of a pulsating magnetic field propagating in the horizontal and vertical directions to neutralize the magnetic field and the following cancel mechanical (Lorentz force), electric and magnetic effects. This system consists of generating magnetic fields by electromagnet connected to a vertical and horizontal movable robot to detect areas of intense electromagnetic noises.

In this context, this paper essentially comprises the following four parts:

- Problematic and objective of the study.

- Design and control of non-neutralizing helpful intense electromagnetic waves.

- Modelling of the neutralizer in the simulation environment Matlab-Simulink.

- Descriptions of the simulations results.

\section{Problem and Aim of the Study}

The propagation of electromagnetic waves is huge special for the excessive use of distance communication means such as internet, mobile and generally radiofrequency and industrial systems. In addition, electromagnetic wave resonances can cause serious problems such as instability of electrical systems, remote electric shocks threatening the life of the human being. These applications provide an undeniable comfort of life, and have many drawbacks which include the essentials:

- Serious threats to the health and vitality of the human being.

- Very serious disturbance of the stability of electric power systems.

- Non-Natural influences on climate.

In this context, this study concerns the design and control of a location, detection and neutralization of non-helpful intense waves. This system is called neutralizer of intense electromagnetic wave. The detecting is assured by a mobile coil for receiving an electromotive force by the traveling wave. This coil is mounted on a mobile robot with high maximum 
speed. This coil is used to identify spatial ordinate of the electromagnetic wave and directs a powerful electromagnet to generate a magnetic induction vector of the same intensity and with opposite direction of the induction vector generated by the electromagnetic wave, which causes its neutralization.

\section{Design of the Electromagnetic Wave Neutralizer}

\subsection{Neutralizer Structure}

The structure of the neutralizer is illustrated in Figure 1. The neutralizer includes a power electromagnet with simple structure to achieve. The slots are straight and open and simpleto achieve. This electromagnet is mounted on a mobile robot moving in the directions of the $\mathrm{x}$ and $\mathrm{z}$ axis (Figure 2). The displacement along the $y$-axis is provided by a linear motor connected to the crawler. The robot is equipped with a position sensor of the intense electromagnetic wave ( $\mathrm{x}, \mathrm{y}$ and $\mathrm{z}$ ) and an intensity of the magnetic induction at this position sensor. Upon detection of the position and the field strength of the magnetic induction of the strong electromagnetic wave, the electromagnet is powered via a DC-DC converter for generating a magnetic field opposite to the magnetic field generated by the intense wave and with same value as this magnetic field, leads to the neutralization of the strongest wave. After neutralization of this electromagnetic wave, robot move according to other intense waves coordinates.

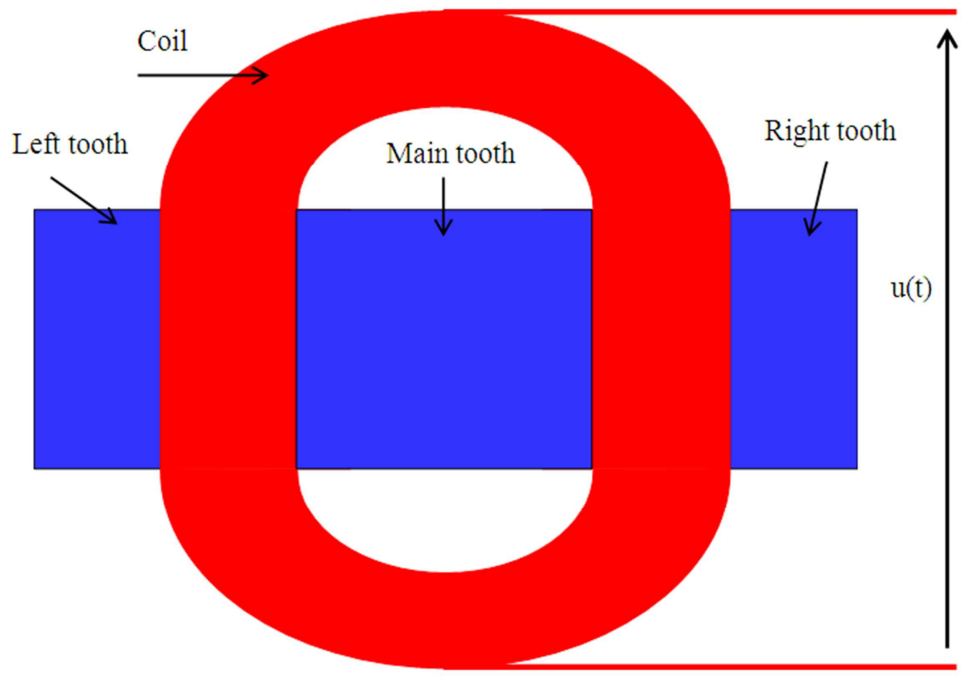

Figure 1. Front view of the electromagnet.

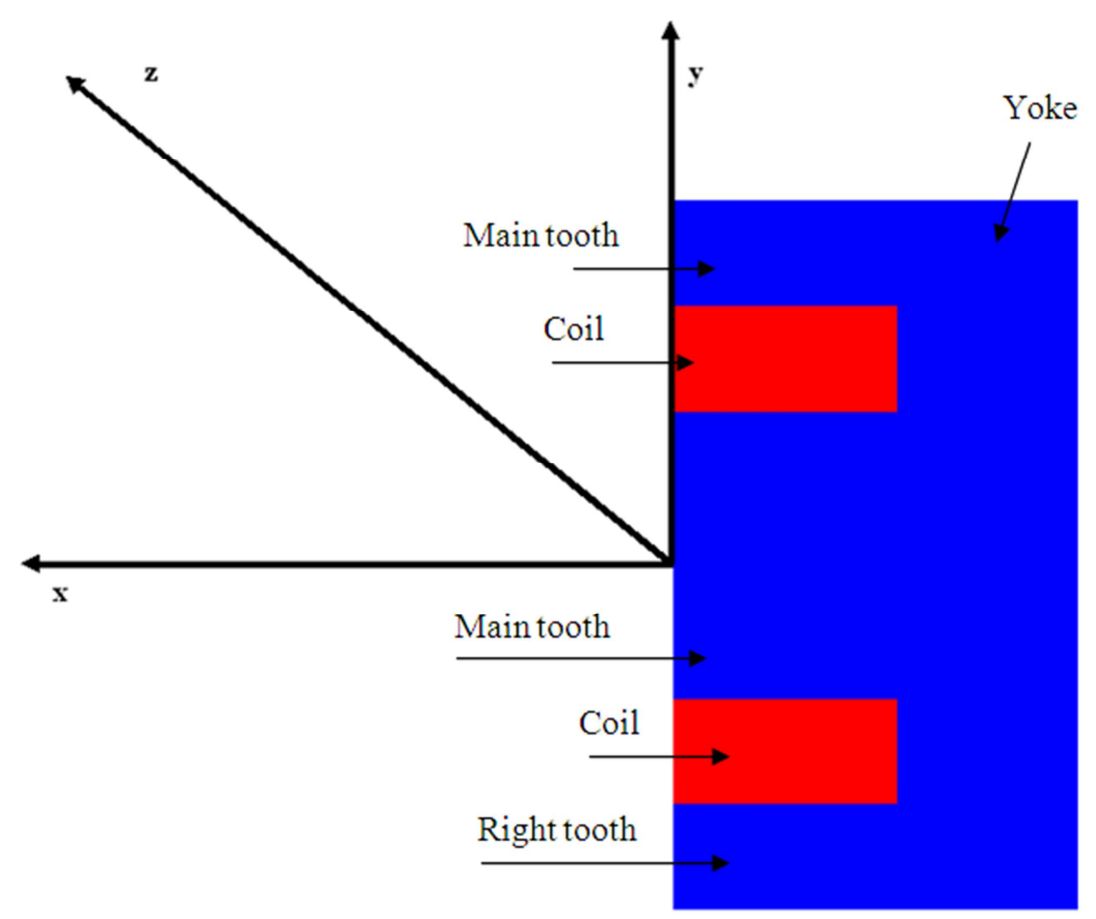

Figure 2. Sectional view of the electromagnet showing the directions of rebot movement. 


\subsection{Sizing and Modelling of the Electro-Magnet}

A design program of electromagnet was developed. This program is primarily supported on the analytical method [1-5].

The main design and modelling equations will be detailed in this paragraph.

The maximum magnetic induction near the teeth is given by the following relationship:

$$
B_{\text {emax }}=\mu_{0} \times \frac{N_{s} \times I_{\text {max }}}{2 \times x_{\text {min }}}
$$

where $\mu_{0}$ is the air permeability, $\mathrm{N}_{\mathrm{s}}$ is the coil number of turns, $\mathrm{I}_{\max }$ is electromagnet maximal current and $\mathrm{x}_{\min }$ distance close to the main tooth.

The section of the main tooth is given by the following relationship:

$$
S_{d m}=L_{m} \times L_{a}
$$

where $\mathrm{L}_{\mathrm{m}}$, La are respectively the length and the width of the electromagnet.

Sections of the right and left teeth are given by the following relationships:

$$
\begin{gathered}
S_{d r}=\frac{B_{e \max } \times S_{d m}}{2 \times B_{d}} \\
S_{d l}=\frac{B_{e \max } \times S_{d m}}{2 \times B_{d}}
\end{gathered}
$$

where $B_{d}$ is the left and right flux density.

The tooth height is given by the following relationship:

$$
H_{d}=\frac{N_{s} \times I_{\max }}{K_{f} \times \delta \times L_{e n c}}
$$

where $\mathrm{Kf}$ is fillingfactor of slots, $\delta$ is the current density and Lenc is the slots width.

The flux density generated by the electromagnet as a function of current and the position is given by the following relationship:

$$
B_{e}(i, x)=\mu_{0} \times \frac{N_{s} \times i(t)}{2 \times x(t)}
$$

where $i(t)$ is the demagnetizing current at $t$ time et $x(t)$ is the position of the electromagnetic wave.

The resistance of the coil is given by the following relationship:

$$
R\left(T_{c}\right)=\frac{N_{s} \times L_{s p} \times \rho\left(T_{c}\right)}{\frac{I_{\max }}{\delta}}
$$

where $L_{s p}$ coil turn longth, $\rho$ is the copper resistivity and $T_{c}$ is the copper temperature.

The inductance of the coil is expressed by the following relationship:

$$
L=\mu_{0} \times\left(\frac{\frac{S_{d m}}{2}}{2 \times x_{\min }}+\frac{H_{d} \times L_{m}}{L_{e n c}}\right) \times N_{s}^{2}
$$

\section{Modelling of the Neutralizer Under Matlab-Simulink}

The neutralizer is modelled under the Matlab-Simulink environnement considering that this simulation environnement provides good accuracy of simulation results and the simulation phase is located just before the practical implementation phase [5-16].

\subsection{Neutralization Flux Density Regulator}

The magnetic induction of neutralization controller (Figure 3 ) receives the coordinates and the intensity of the induction of the electromagnetic wave to the detected position, and allows through a proportional-integral type regulator to generate a signal pulsing at the rate of this wave which is converted via a modulator in a converter control signal [5-16].

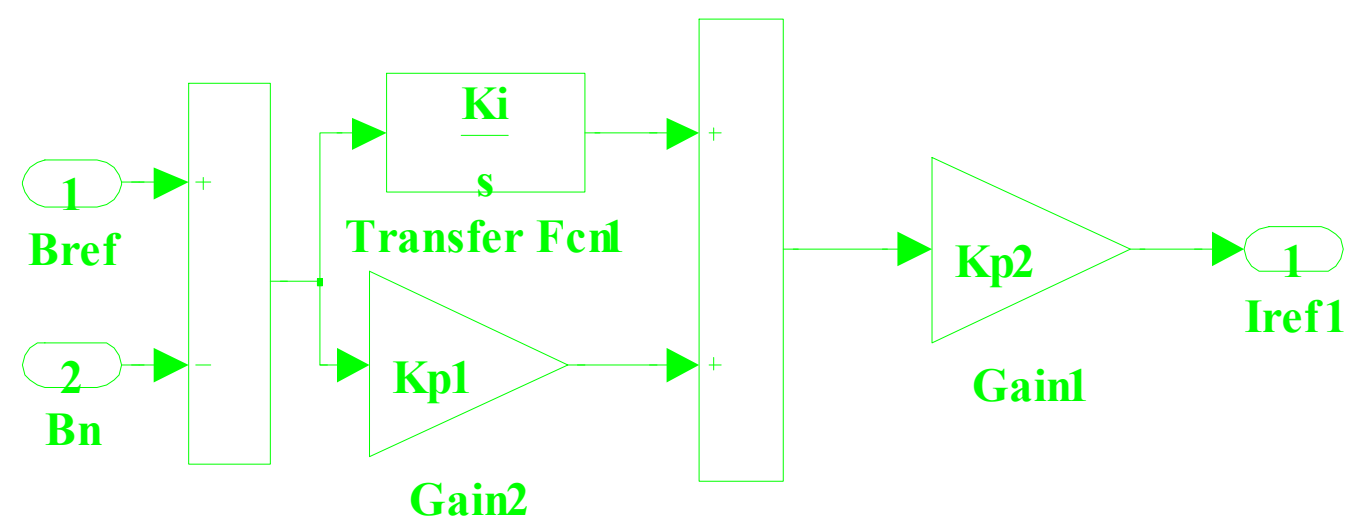

Figure 3. Neutralization flux density regulator. 


\subsection{Control Signal Generator}

The control signal generator (Figure 4) permit to modulate the signal generated by the controller of the intensity of the neutralizing induction of the detected wave by a triangular wave signal. The delay to closing and opening is taken into account by the model [16-17].

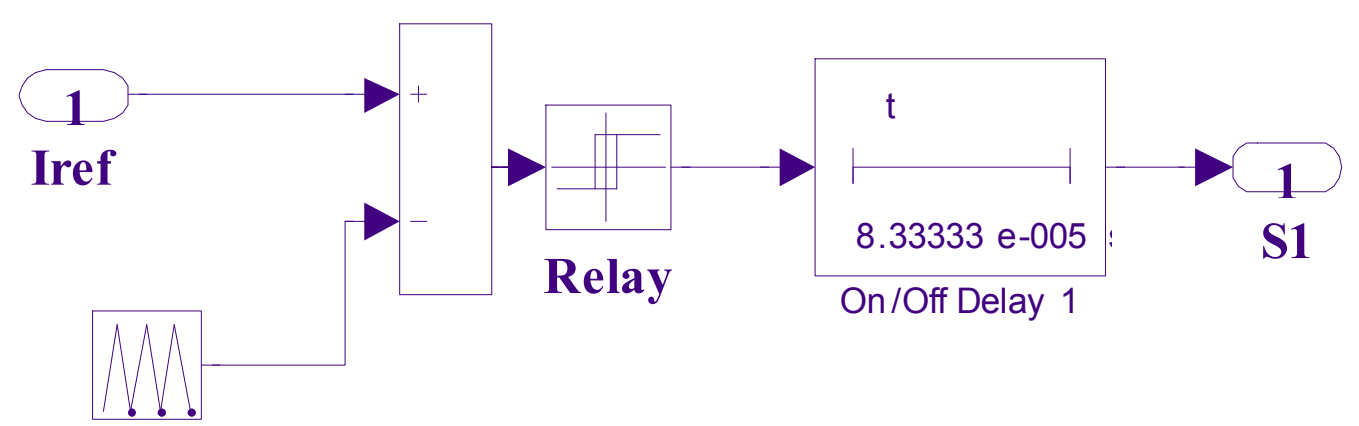

\section{Triangular signal}

Figure 4. Control signal generator.

\subsection{Model of the All Electromagnet-Converter}

The coil of the electromagnet is supplied by a DC-DC converter controlled by the signal generated by the generator of the control signal. The model of the all electro-magnet-converter is shown in Figure 5.

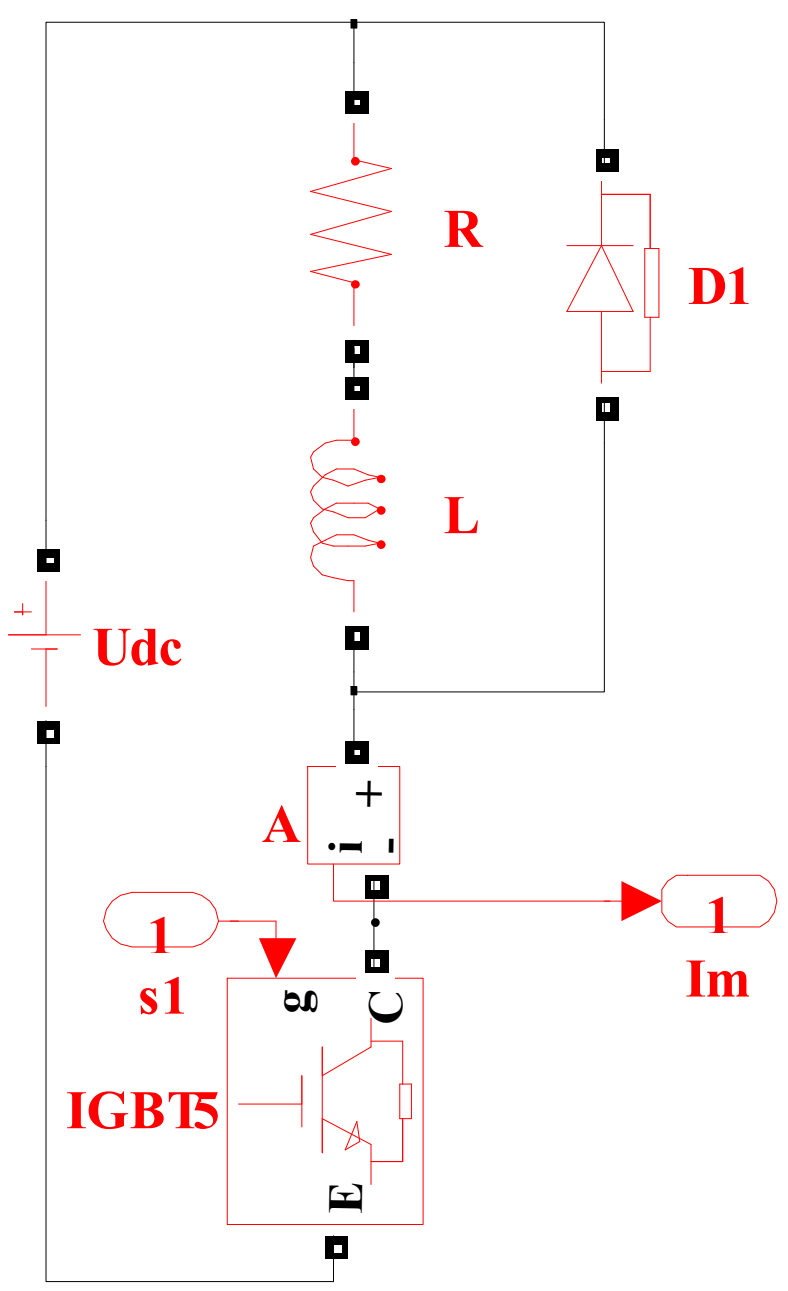

Figure 5. Model of the all electromagnet-converter. 


\subsection{Resulting Flux Density Calculator}

The calculator of the density of the resulting flow (Figure 6) is used to test the entire neutralization of the intense wave.

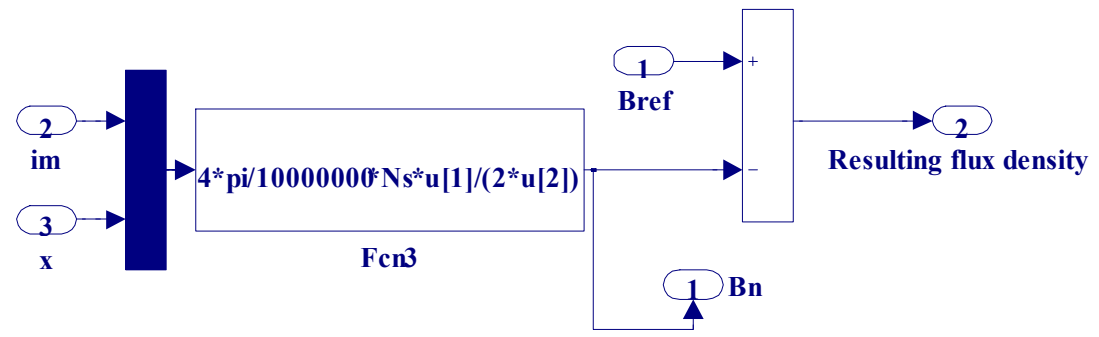

Figure 6. Resulting flux density calculator.

\subsection{Global Model the Neutralizer}

The coupling of different models of the neutralizer, leads to the global Simulink model shown in Figure 7.

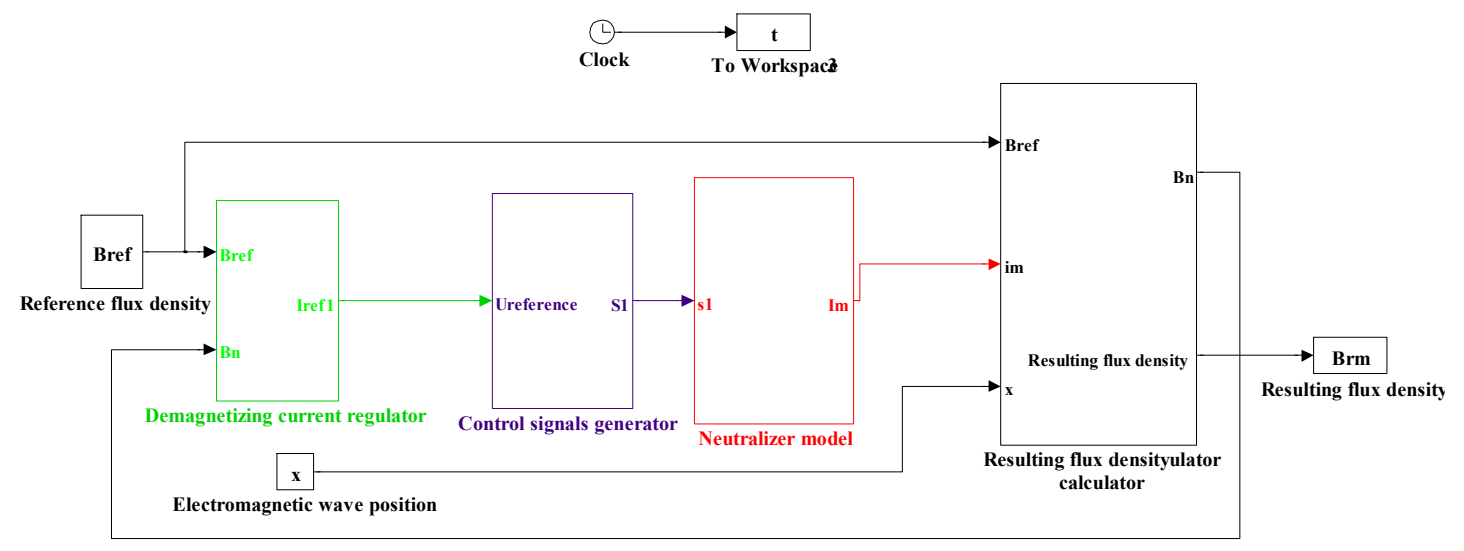

Figure 7. Global model of the neutralizer.

\section{Description of the Simulations Results}

The paces of the supply voltage and the magnetizing current are illustrated in Figure 8. This figure shows the low voltage drop at the supply voltage due to the saturation voltage of the
IGBT transistor. This figure also shows the low switching frequency since the simulation is performed in static. This frequency can be greatly increased for waves propagate with a very high speed.

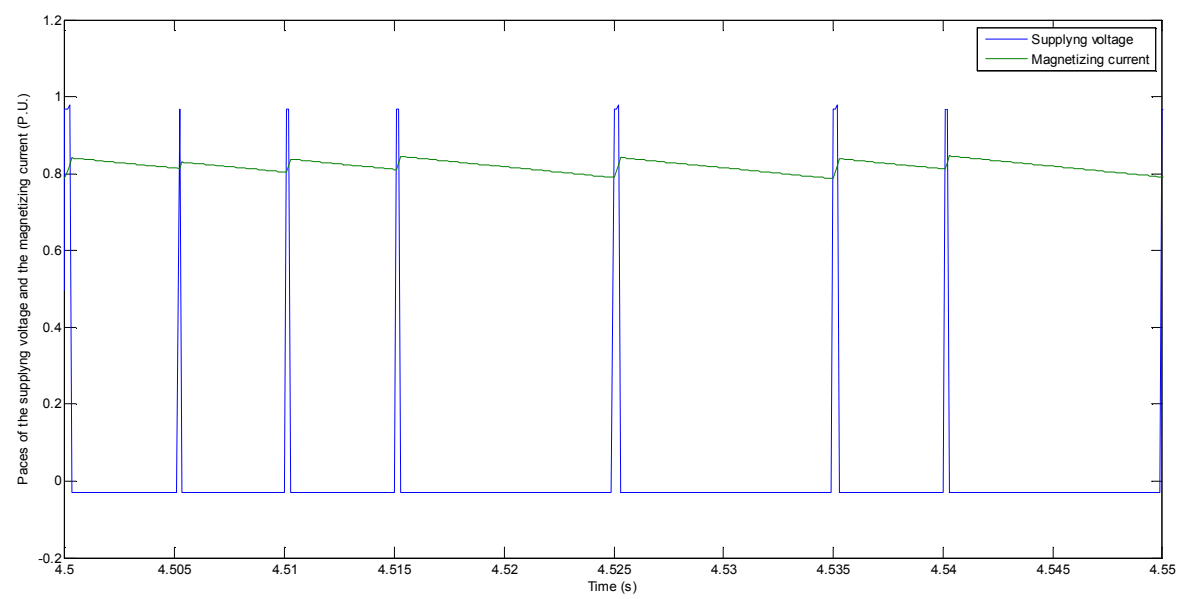

Figure 8. Paces of the supplying voltage and the magnetizing current. 
Figure 9 shows the evolution of the magnetizing current. This current stabilizes after $4 \mathrm{~s}$ confirming that the model will be valid for slow intense waves.

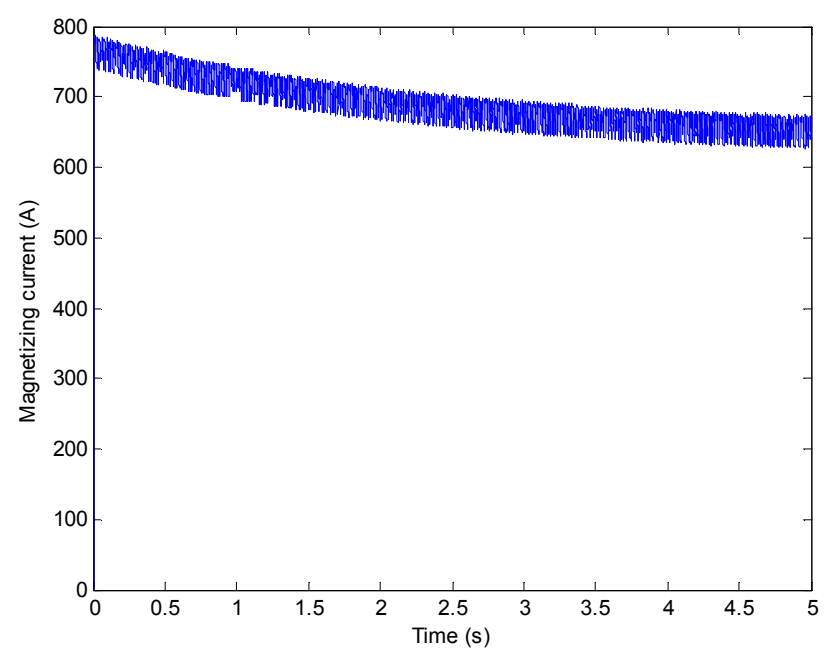

Figure 9. Demagnetizing current evolution.

The Figure 10 Illustrate the Evolution of the reference detected flux density, the magnetization flux density and the resulting flux density. This figure also shows that the developed model is valid for slow intense waves because the neutralization is realised after $4 \mathrm{~s}$.

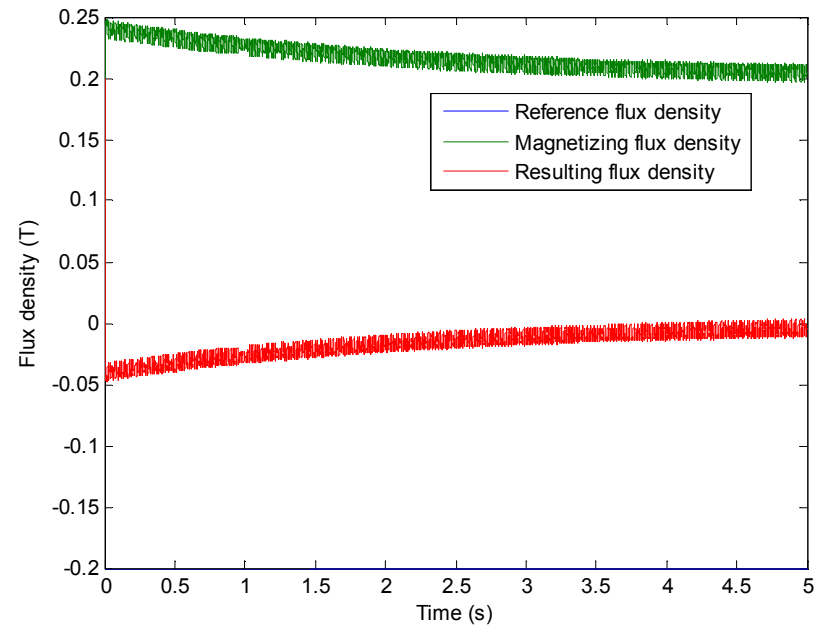

Figure 10. Evolutions of the reference detected flux density, the magnetizing flux density and the resulting flux density.

Finally, simulation results validate the study presented in this paper and opens the avenue of research to extend this study to neutralize a fast intense waves.

\section{Conclusion}

The study presented in this paper concern the design and control of a neutralizer of intense electromagnetic waves. A sizing and modelling program of the neutralizer is developed. This program is validated following its establishment under the simulation environment Matlab-Simulink. Simulation results show that this neutralizer is specialized for slow intense waves. This study can be extended to cover the fast intense waves.

\section{References}

[1] Kacem MH, Tounsi S, Neji R. Losses modeling of the electric vehicles power chain. Int. J. Electr. Compon. Energy Convers. 2015;1:49-54. doi:10.11648/j.ijecec.20150102.11

[2] Gorbel Z, Chihaoui Y, Barg N, et al. Modelling approach of electric cars autonomy. Int. J. Electr. Compon. Energy Convers. 2015;1:55-62. doi:10.11648/j.ijecec.20150102.12.

[3] Tounsi S. Electro-thermal modeling of permanent magnet synchronous motor. Int. J. Electr. Compon. Energy Convers. 2015;1:63-68. doi:10.11648/j.ijecec.20150102.13.

[4] Marouani H., Tounsi S. Design of a coiled rotor synchronous motor dedicated to electric traction. J. Electr. Syst. (JES). 2014;10(3):344-358, Indexed in Scopus.

[5] Tounsi S, Salah IB, Bouhlel MS. Design and control of axial flux Brushless DC motor dedicated to electric traction. J. Autom. Syst. Eng. (JASE). 2014;8(2):62-81.

[6] Tounsi S. Losses modelling of the electromagnetic and IGBTs converters. Int. J.Electr. Hybrid Veh. 2013;5:54-68.

[7] HadjKacem M, Tounsi S, Neji R. Optimization of cutting frequency electromagnetic interuptor. Int. J. Comput. Appl. 2013;67:23-27.

[8] Tounsi S. Comparative study of trapezoidal and sinusoidal control of electric vehicle power train. Int. J. Sci. Technol. Res. (IJSTR). 2012;1(10):99-107.

[9] Kacem MH, Tounsi S, Neji R. Systemic design and control of electric vehicles power chain. Int. J. Sci. Technol. Res. (IJSTR). 2012;1(10):73-81.

[10] Tounsi S. Control of the electric vehicles power chain with electromagnetic switches reducing the energy consumption. J. Electromagnet. Anal. App. (JEMAA). 2011;3(12):537-545.

[11] Tounsi S, Kacem H, Neji R. Design of static converter for electric traction. Int. Rev. Model. Simul. (IREMOS). 2010; 3:1189-1195, Indexed in Scopus.

[12] Tounsi S, Neji R. Design of an axial flux brushless DC motor with concentrated winding for electric vehicles. J. Electr. Eng. (JEE). 2010;10:134-146. Indexed in Scopus.

[13] Tounsi S, Neji R, Sellami F. Design methodology of permanent magnet motors improving performance of electric vehicles. Int. J. Model. Simul. (IJMS). 2009;29(1):96-103.

[14] Chaieb M, Tounsi S, Neji R, et al. Design and optimization of axial permanent magnet machines for electric vehicle. J. Electr. Syst. (JES). 2009;5:17-31, Indexed in Scopus.

[15] Ben Salah B, Moalla A, Tounsi S, et al. Analytic design of a permanent magnet synchronous motor dedicated to EV traction with a wide range of speed operation. Int. Rev. Electr. Eng. (IREE). 2008;3, Indexed in Scopus. 
[16] Neji R, Tounsi S, Sellami F. Contribution to the definition of a permanent magnet motor with reduced production cost for the electrical vehicle propulsion. Eur. Trans. Electr. Power. 2006;16:437-460, Indexed in Thomson reuters.
[17] Begacem A, Fakhfakh MA, Tounsi S. Optimal design and control of electric vehicle power chain. J. Electr. Eng. (JEE), Ed. 2. 2015;15:304-314, Indexed in Scopus. 